Cahiers $d u$ MONDE RUSSE

\section{Cahiers du monde russe}

Russie - Empire russe - Union soviétique et États indépendants

$50 / 4 \mid 2009$

Varia

\title{
Robert Edelman, Spartak Moscow
}

\section{Andreï Kozovoï}

\section{OpenEdition \\ Journals}

Édition électronique

URL : https://journals.openedition.org/monderusse/7197

DOI : $10.4000 /$ monderusse. 7197

ISSN : $1777-5388$

\section{Éditeur}

Éditions de l'EHESS

\section{Édition imprimée}

Date de publication : 15 décembre 2009

Pagination : 857-861

ISBN : 978-2-7132-2261-0

ISSN : $1252-6576$

\section{Référence électronique}

Andreï Kozovoï, «Robert Edelman, Spartak Moscow », Cahiers du monde russe [En ligne], 50/4 | 2009, mis en ligne le 12 janvier 2011, consulté le 03 septembre 2022. URL : http://journals.openedition.org/ monderusse/7197; DOI : https://doi.org/10.4000/monderusse.7197

Ce document a été généré automatiquement le 3 septembre 2022

Tous droits réservés 


\title{
Robert Edelman, Spartak Moscow
}

\author{
Andreï Kozovoï
}

\section{RÉFÉRENCE}

Robert EDELMAN, Spartak Moscow. A History of the People's Team in the Worker's

State. Ithaca - Londres : Cornell University Press, 2009, 346 p.

1 Robert Edelman, professeur à l'université de Californie depuis 1972, spécialiste du sport en Union soviétique et ancien commentateur sportif, nous livre ici une monographie sur l'une des équipes de football les plus connues de Russie, le Spartak de Moscou. L'étude du sport en contexte totalitaire n'est pas nouvelle : après le travail pionnier, à la fin des années 1970, de James Riordan (ancien joueur du Spartak)1, Edelman a écrit, en 1993, un ouvrage de synthèse sur la question ${ }^{2}$. Plus récemment, l'historien russe Mihail Prozumenščikov, l'un des directeurs du RGANI (Archives d'État russes d'histoire contemporaine), a marqué l'historiographie ${ }^{3}$.

2 En neuf chapitres chronologiques, Edelman retrace l'histoire du Spartak, véritable " équipe du peuple» pour ses supporters. Au travers de cette étude de cas, l'auteur se propose d'illustrer tout un pan de vie de la société soviétique et, dans le même temps, d'étudier ses rapports avec le pouvoir. Comprendre les stratégies de contournement du système par les Soviétiques, la fameuse " culture du corps $»^{4}$, la sociologie des joueurs et des publics, la possibilité d'un star system en URSS, tels sont les objectifs de l'auteur.

Le récit s'ouvre par le rappel d'une question provocatrice posée par le diplomate britannique Bruce Lockhart : l'absence d'une ligue professionnelle de football aurait-elle favorisé la révolution en Russie? Interrogation pertinente, puisqu'elle fut posée en sens inverse pour la Grande-Bretagne : le sport fut-il le liant qui maintint soudée la société victorienne ? En Russie comme ailleurs, le football arrive par les marchands britanniques dans les années 1870, notamment par les ports de Saint-Pétersbourg et d'Odessa. Apparu officiellement en 1897, il demeure longtemps anecdotique: en 1912, lors des Jeux olympiques de Stockholm, les performances russes sont désastreuses; à la veille de la Première Guerre mondiale, seulement 8000 joueurs sont enregistrés dans tout l'Empire. 
Ce sont les étrangers qui, jusqu'en 1917, continuent à donner l'impulsion. Ainsi, à Moscou, le rôle de l'ingénieur britannique Harry Charnock à la fabrique textile de Morozov, entre la province de Moscou et Vladimir, est crucial dans l'avènement du futur Spartak. Edelman montre bien comment le football accompagne la naissance d'une culture urbaine, traversée par des vagues d'occidentalisation, dont le football est l'un des vecteurs. Véritable marqueur identitaire, ce sport joue le rôle d'un « compensateur de virilité » aux yeux de la masse paysanne qui voit dans les citadins d'abord des êtres efféminés, habitués au confort.

football russe s'émancipe après 1917, avec le départ des entraîneurs étrangers. Les catégories défavorisées, jusqu'ici exclues, intègrent ce jeu, qui légitime enfin son qualificatif de "sport des masses». Mais la qualité du football russe va pâtir d'une première vague d'émigration; de plus, la propagande sportive le relègue largement derrière les jeux "nobles", hérités des Grecs. Le nouveau pouvoir craint en fait de se laisser dépasser par un sport brutal et imprévisible (chez les joueurs comme chez les spectateurs), d'autant que de nombreux joueurs célèbres sont issus d'un quartier moscovite turbulent, la Presnja, devenue krasnaja (rouge) après 1917, en souvenir de son rôle dans la révolution de 1905. C'est en pleine NEP, dont le contexte de stabilité et de concession aux entreprises lucratives (mais aussi de développement de réseaux criminels en tout genre) permet au football de reprendre son souffle, qu'émerge dans ce quartier le Cercle du sport de Moscou, grâce au talent d'un certain Nikolaj Starostin, attaquant sur le terrain et dans la vie; c'est lui qui, avec ses trois frères, Andrej, Aleksandr et Petr, fondera en 1935 le Spartak de Moscou.

5 Jusqu'au début des années 1950, l'histoire du club est celle d'une institution qui lutte sans cesse pour son professionnalisme et qui, de fait, en pâtit. Patronné par un organe du ministère du Commerce, Promkooperacija - alors que ses ennemis sur le terrain, le Dinamo de Moscou et le CDKA, le sont respectivement par le ministère de l'Intérieur (MVD) et par l'armée -, le Spartak profite de l'impopularité de ces derniers pour devenir un modèle et une institution défouloir pour des centaines de milliers de supporters soviétiques, de plus en plus jeunes, qui hurlent dans l'enceinte du stade: "Mort aux flics! » ou «Mort aux soldats!». En 1937, le Spartak confirme son ascension après sa victoire exceptionnelle sur une équipe basque en tournée en URSS. Cette année constituera aussi un tournant pour les Starostin: leur vie luxueuse, qui tranche avec celle de la majorité des Soviétiques, attise inévitablement des convoitises. C'est le temps des premières dénonciations : selon la rumeur, les Starostin abuseraient de leurs privilèges, favoriseraient le « sport bourgeois »...

6 Mais le pire est à venir: Berija, chargé de la gestion du Dinamo de Moscou, tente d'orchestrer la chute des Starostin en profitant d'une vague de calomnies à leur encontre : ils auraient ourdi le projet d'assassiner Stalin au cours de la parade sportive de 1937 et de créer un "État fasciste» (Edelman s'appuie ici sur des archives de l'armée [RGVA]). Néanmoins, les Starostin ne sont pas arrêtés : selon la légende, Molotov, dont les enfants sont liés aux joueurs, refuse de contresigner l'ordre de Berija. Mais le NKVD ne desserrera plus son étau. Catalogués "droitiers trotskistes ", les frères finissent à la Lubjanka en 1942. Ils sont condamnés à dix ans, peine symbolique pour l'époque. Inexplicablement, les charges retenues sont surtout criminelles. Après des séjours au goulag dans des conditions privilégiées, les quatre Starostin sont libérés en 1954, après la première amnistie. 
7 Pendant le même temps, le pouvoir s'efforce, après-guerre, de mettre l'accent sur l'éducation idéologique et politique des joueurs: plus que jamais, ceux-ci doivent se comporter en modèles. Face à certains radicaux qui réclament des peines exemplaires pour les récalcitrants, le jeune Suslov joue cependant un rôle modérateur. Mais le stalinisme n'en a pas fini avec le football : la défaite soviétique devant les Hongrois en 1952 entraîne de nombreuses répercussions, dont la disparition de l'équipe de l'armée - opportunité sans précédent pour le Spartak. Comme le montre finement Edelman, le contexte d'un premier dégel (sous Stalin!), marqué par le retour du jazz et des danses qui l'accompagnent, profite d'une certaine manière au football, les joueurs intégrant dans leur jeu de nouvelles cadences.

La période qui suit la mort de Stalin est marquée par un long déclin du club, ponctué de plusieurs sursauts. La médaille d'or olympique de 1956 est son chant du cygne. La compétition avec le Dinamo de Moscou est au centre du jeu politique de l'équipe jusqu'en 1961. Après cette date, le monopole disparaît et les clubs locaux se multiplient : de 33 en 1950, on passe à 267 équipes, tous niveaux confondus, en 1968. Le Spartak devient un club parmi d'autres. Même s'il revient au sommet en 1978, son déclin - à en juger par les résultats - est inexorable jusqu'à la fin de la période soviétique. Comment décrypter le fait que cette équipe, en dépit de ses défaites, conserve paradoxalement un grand nombre de supporters fidèles? La principale raison semble en être sa légende dorée de club "démocratique»-même si, en l'occurrence, cet adjectif ne rime pas à grandchose.

L'auteur insiste sur le rôle des pratiques informelles dans les années 1970-1980. Le Spartak use et abuse des « caisses noires » et du blat [régime de faveurs], tandis que se développe la violence dans les stades - reflet d'une agressivité urbaine renouvelée - dont les médias se font l'écho. Edelman clôt sa monographie sur le tournant de 1989-1992, dramatique pour le Spartak comme pour bien d'autres équipes soviétiques en raison de départs massifs de joueurs à l'étranger et d'une privatisation brutale. Il souligne pourtant que les usages acquis depuis l'époque de la NEP se révèlent finalement payants : grâce à son professionnalisme, le Spartak réussira à s'adapter au tournant.

Illustration originale de l'évolution sociale de l'URSS, ce travail fait certes appel à des sources publiées telles que la revue Sovetskij sport, mais aussi à des archives inédites, centrales et régionales : ainsi, pour le GARF, le fonds du Comité de la culture physique et du sport (Gossport SSSR); pour le RGASPI, le fonds du Comité central du PCUS et du Politbjuro ; les archives du RGVA, ainsi que celles de Perm (GAPO). Mais on regrettera que l'auteur ait négligé les sources orales, très nombreuses : ses jugements sur l'opinion des supporters sont le plus souvent des hypothèses ou des généralisations fondées sur des cas particuliers.

11 Le travail aurait aussi gagné en relief si l'auteur s'était penché sur la place du football dans la culture soviétique, par exemple dans la littérature et le cinéma. Mentionnons ici le roman Vratar' respubliki [Le Goal de la république] de Lev Kassil' (1905-1970), le premier du genre, qui eut un tel succès qu'il fut adapté au cinéma (Le Goal, de Semen Timošenko, 1936) ${ }^{5}$. De même, le culte de Spartacus n'est pas abordé : pourtant, le lecteur est en droit de se demander pourquoi le Spartak est le seul club dont le nom honore un « dirigeant révolutionnaire du passé » (p. 74).

Plus encore, pour une recherche qui s'inscrit dans l'histoire sociale soviétique, un plan thématique, et non chronologique, aurait été préférable. Pour prendre un seul exemple, en quoi l'année 1953 constitue-t-elle une rupture pertinente pour l'histoire du Spartak, 
Stalin, comme l'écrit lui-même l'auteur, n'ayant jamais été un fervent admirateur du football ? Le choix du plan est sans doute dicté par des impératifs éditoriaux autant que par la volonté de contextualiser l'histoire du football et du Spartak en particulier, mais cette dernière se révèle largement stérile, tant le lien entre contexte politique intérieur et international est ténu ( $a u$ vu des archives disponibles). Par ailleurs, l'histoire du club verse très souvent dans l'anecdotique. Les nombreuses illustrations, rarement commentées, ainsi que certaines annexes ${ }^{6}$, témoignent d'une absence de rigueur scientifique dans l'approche du sujet.

Le travail de Robert Edelman souffre d'un écueil presque inévitable : c'est l'œuvre d'un supporter qui ne parvient pas à donner à sa passion une dimension pleinement scientifique et à transcender ce qui est d'abord de l'ordre de l'émotionnel. Le parti pris de l'auteur transparait aussi dans le choix des auteurs cités à longueur de pages et dont la pertinence est souvent douteuse. Outre Bourdieu, véritable marqueur idéologique en sciences sociales, Edelman émaille son récit de citations d'autorités telles que Moshe Lewin. De fait, le style de l'auteur apparaît très « lewinien », marqué par de nombreuses lourdeurs et digressions qui font perdre le fil de l'ensemble.

Un plan thématique aurait aussi permis d'éviter l'écueil trop fréquent d'un déséquilibre entre les parties : si le chapitre III porte sur 1936-1937, le chapitre IX, lui, balaie les années 1964-1991 qui auraient pourtant mérité un traitement plus approfondi. On sait par exemple que Konstantin Černenko était un grand supporter du Spartak de Moscou, et qu'il fit tout son possible pour améliorer la situation du club après sa dégringolade de $1976^{7}$. Edelman aurait également pu établir un parallèle entre les attitudes favorables du public envers le Spartak et celles à l'égard de Garri Kasparov, joueur d'échecs à la réputation de " démocrate ", qui affronte au milieu des années 1980 un Anatolij Karpov, favori des apparatchiks. De même, des chapitres thématiques auraient permis de mettre en valeur un aspect important de l'histoire du Spartak - les déplacements (des joueurs et du public), en URSS et surtout à l'étranger. Comment fut sélectionné et conditionné le public des supporters du Spartak lors de ses tournées en Occident ? Ces séjours avaientils une quelconque incidence sur ce même public?

En dépit de ces remarques, il convient de souligner que la monographie de Robert Edelman mérite toute sa place dans la nouvelle historiographie de l'histoire de l'URSS, en ce qu'elle révèle la dimension de l'imprévisible dans un monde où tout semble écrit à l'avance pour l'ensemble des Soviétiques, du berceau à la tombe.

\section{NOTES}

1. Sport in Soviet Society: Development of Sport and Physical Education in Russia and the USSR, Cambridge, MA : Cambridge University Press, 1977.

2. Serious Fun: a History of Spectator Sports in the USSR, Oxford : Oxford University Press, 1993.

3. Bol'šoj sport $i$ bol'šaja politika [Le grand sport et la grande politique], M. : Rosspen, 2004.

4. Robert Edelman fait siennes les conclusions de Henning Eichberg dans Body Cultures: Essays on Sport, Space and Identity, Londres : Routledge, 1998. 
5. De fait, pour le cinéma, auraient mérité d'être traités comme illustrations d'une culture footballistique en évolution des longs métrages tels que L'Honneur sportif (1951, Vladimir Petrov) ; Le Remplaçant (1954, Sergej Timošenko); Troisième mi-temps (1962, Evgenij Karelov) ; Mon meilleur ami, le général Vassili, fils de Joseph (1991, Viktor Sadovskij).

6. L'annexe III figure un hall of fame (une sélection des meilleurs joueurs du Spartak, selon l'auteur).

7. Voir les Mémoires de l'adjoint de Černenko, Viktor V. Pribytkov, Apparat : 390 dnej i vsja žizn' Genseka Černenko [L'appareil : 390 jours et toute la vie du secrétaire général Černenko], M. : Molodaja Gvardija, 2002. 